\title{
Crack growth based life prediction approach under LCF-HCF interaction
}

\author{
A. Sarkar, A. Nagesha, R. Sandhya \\ Fatigue Studies Section, Metallurgy and Materials Group, Indira Gandhi Centre for Atomic Research, Kalpakkam, Tamil \\ Nadu, India-603102 \\ aritra@igcar.gov.in, bttps://orcid.org/0000-0002-8438-320X \\ nagesh@igcar.gov.in,https://orcid.org/0000-0002-2025-8100 \\ san@igcar.gov.in

\section{Okazaki} \\ Department of Mechanical Engineering, Nagaoka University of Technology, Japan-940-2188 \\ okazaki@mech.nagaokaut.ac.jp, bttps://orcid.org/0000-0001-7071-0399
}

ABSTRACT. Prediction of cyclic life under low cycle fatigue - high cycle fatigue (LCF-HCF) interaction is of paramount importance in the context of structural integrity of components in the primary side of fast reactors where such damage under LCF-HCF interaction occurs. The present investigation deals with the crack growth behavior of a type 316LN austenitic stainless steel subjected to simultaneous application of LCF and HCF cycles (blockloading). Tests were performed over a wide range of temperatures from ambient to $923 \mathrm{~K}$. Experimental results indicate that a critical crack-length $\left(a_{c r}\right)$ exists, beyond which the LCF-HCF interaction becomes significant. An attempt was made to predict life under block cycling by estimating the $a_{c r}$ using fatigue crack threshold $\left(\Delta K_{t b}\right)$ since the latter is known to be affected significantly by the loading history. A universal equation, based on the concept of an equivalent critical crack length $\left(a_{c r, e q}\right)$ which incorporates the damage contribution from DSA and ratcheting under combined LCF-HCF loading, was proposed for life estimation..

KEYwORDS. LCF; HCF; LCF-HCF interaction; Crack growth; 316LN SS.

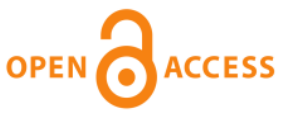

Citation: Sarkar, A., Nagesha, A., Sandhya, R., Okazaki, M., Crack growth based life prediction approach under LCF-HCF interaction, Frattura ed Integrità Strutturale, 50 (2019) 86-97.

Received: 26.11 .2018

Accepted: 24.06 .2019

Published: 01.10.2019

Copyright: (C) 2019 This is an open access article under the terms of the CC-BY 4.0, which permits unrestricted use, distribution, and reproduction in any medium, provided the original author and source are credited.

\section{INTRODUCTION}

$\mathrm{M}$ ost of the current investigations pertaining to the fatigue behavior of structural materials are dedicated to either low cycle fatigue (LCF) or high cycle fatigue (HCF) loading even though it is a well known fact that engineering components experience a varying load history (interaction between LCF and HCF) throughout their service life. Currently, this is a significant issue in sodium-cooled fast reactors (SFRs) where components of the primary sodium circuit are prone to damage induced by LCF as well as HCF which can lead to a significant reduction in 
service life of such components [1-2]. Prediction of fatigue life under LCF-HCF interaction is thus highly essential to ensure the integrity of the components. In this regard, the well-accepted Miner's linear damage summation rule (LDR) poses serious non-conservatism in terms of huge deviations from linearity due to the large difference in lives between LCF and HCF [3-4]. LDR is further refined by modeling attempts, leading to "Damage Curve Approach" (DCA) [5]. However, a major limitation of DCA is its semi-empirical nature which does not account for some intrinsic factors like crack length. Such disadvantages will become more prominent at extreme conditions such as elevated temperature LCFHCF interactions. This necessitates the development of alternate life-prediction models based on crack propagation with periodic measurement of crack length. In view of this, the block-loading experiments including combinations of both LCF and HCF are specially designed so that crack-growth based life-prediction models can be developed based on the same.

\section{EXPERIMENTAL METHODOLOGY}

$\square$ ylindrical fatigue specimens with a semi-circular initial surface notch of $100 \mu \mathrm{m}$ diameter and $50 \mu \mathrm{m}$ depth (Fig. 1) were chosen to study fatigue crack growth behavior under block-loading (as per Fig. 2) at $573 \mathrm{~K}$. Cumulative fatigue damage in terms of LCF-HCF interaction occurs in sodium-cooled fast reactor (SFRs). A closer simulation of the actual reactor conditions can be realized through a loading pattern involving repeated blocks consisting of LCF as well as HCF cycles. Since LCF stress/strain for every start-up and shut-down operation actually results in one cycle and HCF stress/strain are caused by in-service vibrations, the loading pattern in a block shown in Fig. 2 consists of one fully reversed LCF cycle followed by a specific number of HCF cycles superimposed on the LCF cycle which introduces mean stress/strain during HCF cycling. Short fatigue crack growth rate was reported to increase with increase in notch-tip radius [6]. Similar effect was found to follow in case of notch-sensitivity [7]. In the present investigation, the main reason for using a small surface notch was to enable LCF-HCF interaction under block-loading at higher temperatures avoiding extensive intergranularity or tendency towards rupture which is accounted for by creep, ratcheting and their interactions with LCF or HCF. The block-loading experiments are hence redesigned to ensure that specimen fails through initiation and propagation of cracks rather than rupture, so that crack based life-prediction models can be applied to them.

The combined cycling experiments on notched specimens were designed in two steps on similar lines as carried out on smooth specimens in earlier investigations [8]. In Step-I, the specimens were initially subjected to strain controlled LCF loading up to a specific number of cycles corresponding to stabilization of the cyclic stress response (CSR). However, unlike smooth specimen testing, in the present case, the LCF cycling in Step-II was carried out under strain control $\left(\Delta \varepsilon_{\mathrm{t}} / 2_{\mathrm{LCF}}: \pm 0.6 \%\right)$ and not under stress-control to prevent tendencies of rupture through strain accumulation induced by creep and creep assisted ratcheting. HCF cycles of three different $B_{s}(1,10$ and 200$)$ with strain amplitude $\left(\Delta \varepsilon_{\mathrm{t}} / 2_{\mathrm{HCF}}\right)$ of $\pm 0.1 \%$ ( $\sigma_{\mathrm{HCF}}$ of $150 \mathrm{MPa}$, using elastic modulus) were introduced at the maximum LCF strain (Fig. 2), once the CSR reaches stabilization. $B_{s}$ indicates the ratio of HCF cycles to LCF cycles in a particular block. The notch was considered as the initial crack and propagation of the crack from the notch was studied by taking replicas of the specimen surface after interruption at regular intervals, particularly in the short crack growth domain. In the latter stages of crack growth, travelling microscope was used to measure the crack length.

In the present study carried out under strain-controlled mode, significant mean strain will act on the specimen which will lead to plastic ratcheting ahead of the crack tip. This phenomenon was considered for developing the life-prediction model. The model was further refined by carrying out block-loading experiments at higher temperatures, in the range from 573 to $923 \mathrm{~K}$ at a high $\mathrm{B}_{\mathrm{s}}$ of 5000 .

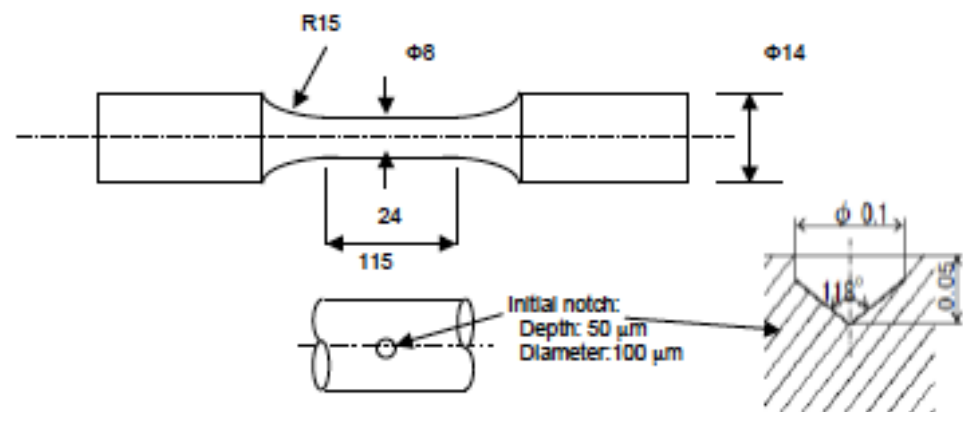

Figure 1: Geometry of the specimens used for crack growth experiment 


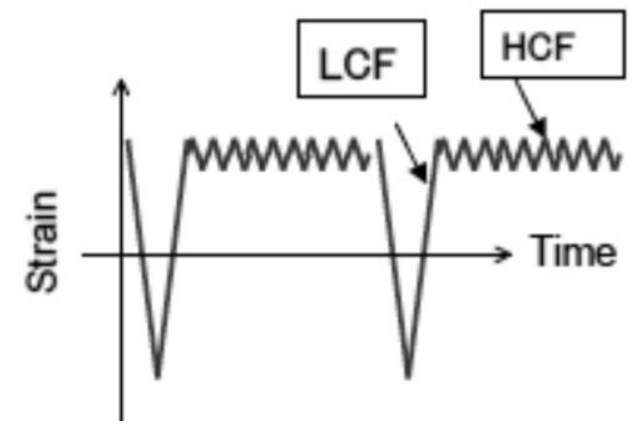

Figure 2: Block loading sequence under strain control used in the crack growth experiments.

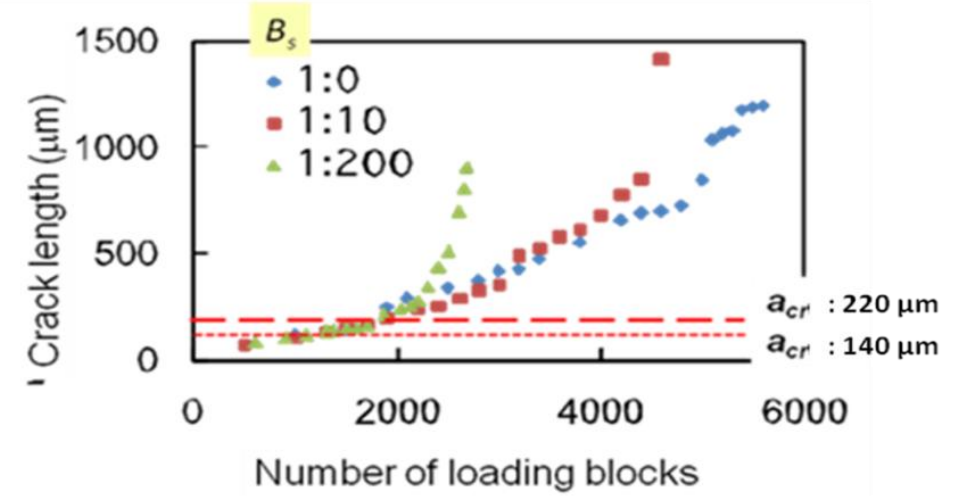

Figure 3: Fatigue crack propagation behavior under LCF-HCF interaction with different $B_{s}$ under strain control with $\Delta \varepsilon_{t} / 2_{L C F} \pm 0.6 \%$ and $\Delta \varepsilon_{t} / 2_{H C F}: \pm 0.1 \%$ (Equivalent $\Delta \sigma_{H C F}: 150 \mathrm{MPa}$ ) T: $573 \mathrm{~K}$

\section{RESULTS AND DISCUSSION}

\section{Establishing a critical criterion for occurrence of LCF-HCF interaction}

$\mathrm{F}$ ig.3 presents the variation of crack length with the number of loading blocks at $573 \mathrm{~K}$, under a loading sequence given in Fig. 2. It is observed that the crack propagation was similar irrespective of the $\mathrm{B}_{\mathrm{s}}$ till a 'critical' crack length $\left(a_{\mathrm{cr}}\right)$ was attained. However, once the crack length extends beyond $a_{\mathrm{cr}}$, significant acceleration in the rate of crack growth was noted, depending on the $B_{s}$. The rate of crack propagation is found to be highest for a higher $B_{s}$ of 200 compared to the lower $\mathrm{B}_{\mathrm{s}}$ of 10 and 1 (only LCF cycling). This resulted in the shortest fatigue life in the former case $\left(\mathrm{B}_{\mathrm{s}}\right.$ : 200) amongst all the three $B_{s}$ used in the present study. It may be noted that $B_{s}$ is an important variable in Fig. 3. The concept of "critical crack length" is based on the fact that the crack growth data coincides in a similar manner irrespective of the $B_{s}$, till a particular crack length is reached, beyond which significant difference in crack growth data is observed with variation in $B_{s}$. According to Miner's rule, the damage under the HCF cycles in the block is considered as zero when the stress amplitude in HCF is much below the fatigue limit and hence cannot impart any significant damage. In the present case, since the HCF strain amplitude is very low $( \pm 0.1 \%)$, equivalent $\sigma_{\mathrm{HCF}}$ is calculated using elastic modulus at $573 \mathrm{~K}$ for nuclear grade 316LN SS (0.07wt.\%). At $573 \mathrm{~K}$, E value for nuclear grade 316LN SS (0.07wt.\%) was reported as $1.5 \mathrm{GPa}$ [9]. Using a strain amplitude of $\pm 0.1 \%$, equivalent $\sigma_{\mathrm{HCF}}$ is estimated as $150 \mathrm{MPa}$ which was less than the fatigue limit [1011]. The strain-ratio $(R)$ during the HCF cycling is 0.71 . However, the crack is found to propagate even during the HCF cycles, once $a_{c i}$ is reached, as reflected from the change in crack growth behavior with respect to $B_{s}$ (Fig. 3 ) indicating that Miner's hypothesis may be untrue for the present experimental results. This suggests that even though the HCF stress is far below the fatigue limit, HCF cycling contributes significantly to the crack growth, once the length of the crack (which may be nucleated from the LCF cycle) reaches the critical value of $a_{c r}$. In other words, strong LCF-HCF interaction prevails beyond $a_{c r}$. It may be possible that crack-growth data overlap at low number of cycles (blocks). However, it may be noted that the present tests are carried out at fixed LCF strain amplitude of $\pm 0.6 \%$, varying only the HCF cycles superimposed on it $\left(B_{s}\right)$. This suggests that crack growth data should be similar for all the cases unless the HCF cycling imparts considerable influence on the crack growth. Hence, the difference incurred in the crack growth data is accounted 
to the variation in $B_{s}$ only. Moreover, in the present case, the similitude in the crack growth data was observed upto 2000 blocks for all the three different $B_{s .}$ It is important to note that 2000 blocks constitute up to almost $30 \%$ of life for $B_{s}: 1$ ( $B_{\mathrm{s}}$ $: 1$ indicates cyclic loading under strain amplitude of $\pm 0.6 \%$ without any HCF cycles). On the other hand, 2000 blocks constitute upto almost $60 \%$ of life for $B_{\mathrm{s}}: 200\left(B_{\mathrm{s}}: 200\right.$ indicates cyclic loading under strain amplitude of $\pm 0.6 \%$ with 200 HCF cycles per each LCF cycle). Clearly, overlapping of crack growth data is possible up to such a large extent of fatigue life. This confirms the previous argument and clearly brings out the importance of $B_{s}$ and the concept of $a_{c r}$ in the crack growth behavior under the superimposed loading pattern as used in the present case. Once $a_{c r}$ is reached, further crack growth is facilitated by LCF as well as the HCF cycling which significantly curtails the fatigue life. This is aptly reflected from the variation in fatigue life presented in Fig. 3 with increase in $B_{s}$. The above point is also corroborated through smooth specimen tests exposed to sequential LCF and HCF cycling where a critical damage marking LCF-HCF interaction was found to occur depending on the degree of LCF pre-exposure and magnitude of LCF strain amplitude employed [12]. This is explained on the basis that at least one Stage-II crack should initiate during the LCF pre-cycling which will grow further during the subsequent HCF phase [13]. The crack-growth experiments depicted in the present study show similar result where the crack will grow at an accelerated rate under the influence of HCF only when $a_{c r}$ is reached. As observed from Fig. 3, $B_{s}$ is an important variable which affects the crack propagation rate. However, the concept of "critical crack length" is based on the fact that the crack growth data coincides in a similar manner irrespective of the $B_{s}$, till a particular crack length is reached. In other words, the critical crack length $a_{c r}$ is not affected by the variation in $B_{s}$. Crack propagation under HCF (minor) cycling is possible only when $a_{c}$ is reached, following which a change in crack growth behavior with respect to $B_{s}$ is observed.

Based on the principle of fracture mechanics, no crack propagation can take place when $\Delta K$ is $<\Delta K_{t b}$ [14]. In the present case, crack propagation (Stage-II crack) commences once the critical crack length $a_{c r}$ is reached. Hence, $\Delta K_{t h}$ can be correlated with $a_{c r}$ using a mathematical relation between $\Delta K$ and $a$, as shown in Eqn. 1. This expression can be utilized for the estimation of $a_{c r}$.

$$
a_{c r}=\left\{\Delta K_{t b} / \quad\left(F \Delta \sigma_{H C F} \sqrt{ } \pi\right)\right\}^{2}
$$

where $\mathrm{F}$ is the boundary correction factor associated with the stress intensity factor and is a function of the shape of the crack. For a semi-circular shaped crack (as in the present case), $\mathrm{F}$ is typically considered as 0.64. Since $a_{c r}$ also marks the onset of LCF-HCF interaction and no such interaction takes place below $a_{c r}$, estimation of $a_{c r}$ using the above expression is an important criterion for life-prediction under LCF-HCF interaction. The crack length may not remain fully semi-circular throughout the process of crack growth. However, this may not affect $a_{c r}$ since $a_{c r}$ is independent of $B_{s}$ and further crack propagation commences only after $a_{c r}$ is reached. Hence, usage of SIF for a semi-circular shaped crack is quite reasonable in the present case.

The threshold stress intensity factor, $\Delta K_{t h}$ is found to be a strong function of the stress ratio, $R$ [15-16]. $\Delta K_{t h}$ shows a gradual decrease with increase in $R$ followed by saturation [14-15]. The value of $R$ was kept at 0.71 in the present blockloading experiments. Although $\Delta K_{t b}$ is usually computed from stress-controlled experiments, in the present case, $\sigma_{H C F}$ is used for $\Delta K_{t h}$ calculation. [17]. The $\Delta K_{t h}$ value at $\mathrm{R}=0.7$ on nuclear grade $316 \mathrm{LN} \mathrm{SS}$ austentitc stainless steel $(0.07 \mathrm{wt} . \%)$ at ambient temperature was reported by Samuel at al. as $\sim 6 \mathrm{MPa} ل_{\mathrm{m}}[15]$. However, with increase in temperature, there is a further reduction in the $\Delta$ Kth value, as reported by Okazaki et al [17]. $\Delta K_{t b}$ was determined experimentally using a $\Delta K$ decreasing fatigue crack propagation test according to the ASTM standard [18] at $R=0.7$. Additionally, $\Delta K_{t b}$ was estimated using at $R=0.7$ using the linear variation of $\Delta K t h$ with temperature as suggested by Shih et al. [19]. Using both the methodologies, $\Delta K_{t b}$ was determined in the band of 3-4 MPa $\sqrt{\mathrm{m}}$, at $573 \mathrm{~K}$ with $\mathrm{R}=0.7$. The authors have used these values of $\Delta K t h$ for estimation of $a_{c r}$

Then, $a_{c r}$ is approximately estimated using Eqn. 1 as follows:

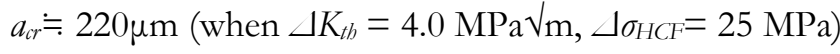

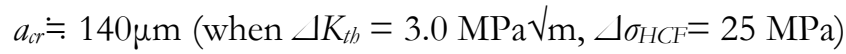

These values are displayed in dotted lines in Fig. 3. This indicates that the prediction is more reasonable compared to the traditional Miner's rule. Hence, in practice, it is essential to ensure that the crack length remains below $a_{c r}$ so as to preempt the occurrence of significant LCF-HCF interaction.

\section{Mechanisms of LCF-HCF interaction}

Crack growth behavior under LCF is characterized by a quick transition from Stage-I to Stage-II crack followed by StageII crack growth over the majority of fatigue life. On the other hand, the bulk of the fatigue life is spent in Stage-I under 
HCF where the transition from Stage-I to Stage-II crack gets much delayed. Similar behavior is corroborated through fatigue crack growth experiments conducted on 316L SS by Miyahara et al [20] which clearly showed that fatigue crack propagation starts much early at cycle-ratio of 0.1 to 0.2 at high strain amplitudes of $\pm 1.0 \%$ or $\pm 0.6 \%$ compared to higher cycle ratios of 0.6 to 0.7 for low strain amplitudes of $\pm 0.3 \%$ or $\pm 0.2 \%$. This above behavior can be also followed from the relationship between fatigue crack growth and strain amplitude under FM expressed as

$$
d a / d N=A\left(\Delta \varepsilon_{i n}\right)^{\mathrm{n}} a
$$

where $d a / d N=$ crack propagation rate, $\Delta \varepsilon_{i n}=$ inelastic strain, $a=$ instantaneous crack length and $n \& A=$ material constant. The experiments in the present case are carried out on notched cylindrical specimens under strain control where the crack tip plasticity is quite high. So, the stable crack propagation behavior is governed by a non-linear fracture mechanics parameter $\Delta J$ rather than $\Delta K$. Dowling et al. [21] and El Haddad et al. [22] have earlier demonstrated a methodology of using J-integral concept as an elastic-plastic fracture mechanics criterion for predicting crack growth behavior. Similar concepts were put forth by Starkey et al. [23] and Haigh et al [24] where crack growth under high strain conditions was considered. The stress-based crack growth equation which is primarily related to LEFM concepts were suitably modified to demonstrate a smooth transition between crack growth rates under high strain conditions involving significant plasticity and that under LEFM conditions involving mostly elastic behavior [23]. Starkey et al [23] also derived a methodology for computing strain intensity factor using half the elastic plus the plastic strain range

Eqn. 2 used in the present case can be derived as follows:

$$
d a / d N=C(\Delta J)^{m} \text { with } \mathrm{m}=1 \ldots
$$

Also,

$$
\Delta J=\Delta K^{2} / E+f(n) \Delta \sigma \Delta \mathcal{E}_{\text {in }} a=\Delta K^{2} / E+f(n) B\left(\Delta \mathcal{E}_{\text {in }}\right)^{1+n} a
$$

using the cyclic stress-strain relationship of $\Delta \sigma=B\left(\Delta \mathcal{E}_{i n}\right)^{n}$

Hence [16],

$$
\Delta \mathrm{J} \simeq f(n) B\left(\Delta \varepsilon_{i n}\right)^{n+1} a \ldots
$$

where

$$
f(n)=\left\{3.85(1-n) / \sqrt{ }_{n}\right\}+\prod n \ldots \ldots
$$

$f(n)$ is computed using a value of ' $n$ ' as 0.2 which is typical value of cyclic strain hardening exponent in stainless steel [22]. Then, equating both $2 \mathrm{a}$ and $2 \mathrm{~b}$, the expression for Eqn. 2 can be derived.

It may be noted that this equation does not cover the crack initiation life, $N_{i}$ and remains valid mostly for Stage-II crack propagation. $N_{i}$ is negligibly small enough under LCF (limited to lower cycle-ratios), however, the same is quite significant in the HCF (higher cycle-ratios). Hence, $N_{i}$ needs to be accounted for when LCF-HCF interaction is concerned.

To utilize this concept under LCF-HCF interaction, crack growth behavior is studied under strain control mode at strain amplitudes $\pm 0.6 \%$ (LCF) and $\pm 0.1 \%$ (HCF), at $573 \mathrm{~K}$ (Fig. 4). It is clear from Fig. 4 that crack propagation is considerably delayed to higher cycle-ratios in case of HCF as compared to LCF. However, an interruption in the crack growth test under LCF at a given cycle-ratio followed by HCF loading condition will lead to significant LCF-HCF interaction which will change the crack growth behavior (indicated by the arrow in the figure). It is clear from the figure that such sequence $(\mathrm{L}-\mathrm{H})$ as indicated by the arrow will significantly curtail the domain of short crack growth under HCF, thereby shortening the crack propagation under HCF. This is the essence of LCF-HCF interaction which leads to drastic fall in remnant HCF lives with prior LCF cycling.

\section{Development of a unified model for life-estimation under block-loading at different temperatures}

It is worthwhile to examine whether the method of life-estimation which has been derived from the block-loading experiments conducted at $573 \mathrm{~K}$, can be extended to other temperatures as well, in the range 573-923 K where damage contributions like plastic ratcheting, creep and creep-assisted ratcheting become noticeable. 


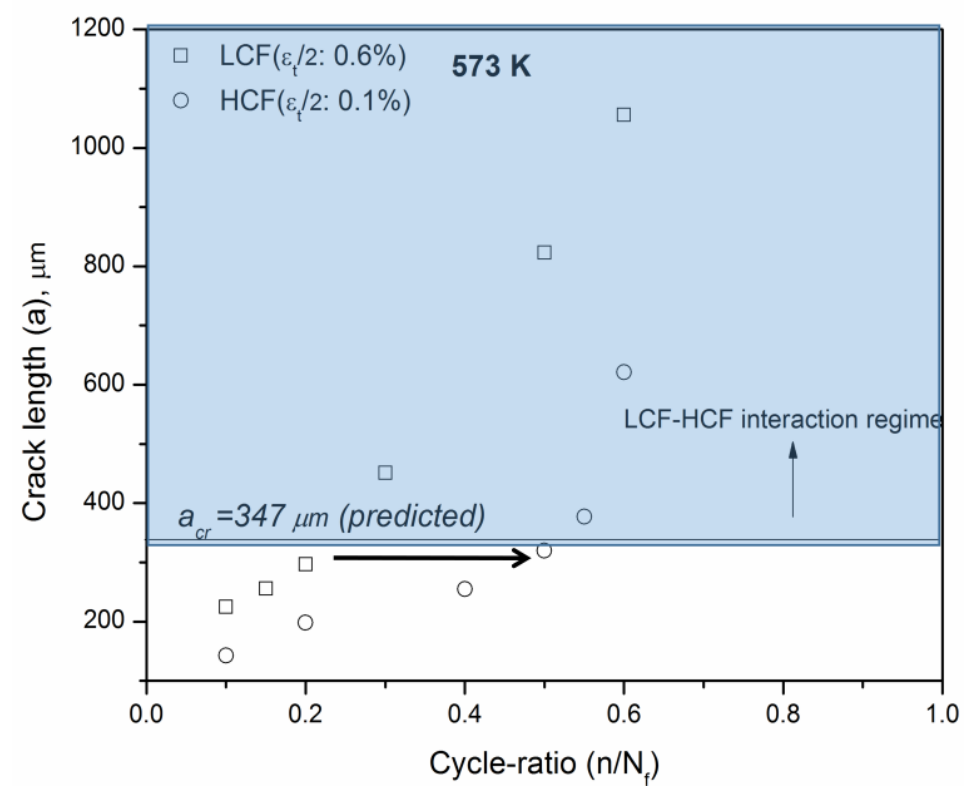

Figure 4: Crack growth behavior at $\Delta \varepsilon_{\mathrm{t}} / 2_{\mathrm{LCF}}: \pm 0.6 \%$ and $\Delta \varepsilon_{\mathrm{t}} / 2_{\mathrm{HCF}}: \pm 0.1 \%, \mathrm{~T}: 573 \mathrm{~K}$. Predicted value of a $\mathrm{a}_{\mathrm{cr}}$ is marked in the figure. The arrow indicates possible LCF-HCF interaction through a sequence of LCF followed by HCF.

As indicated earlier, the crack propagation behavior under a given strain amplitude can be expressed through the following mathematical relation:

$$
d a / d N=\mathrm{A}\left(\Delta \varepsilon_{\text {in }}\right)^{\mathrm{n}} \text { a }
$$

where $d a / d N=$ crack propagation rate, $\Delta \varepsilon_{i n}=$ inelastic strain range, $a=$ instantaneous crack length and $\mathrm{n} \& \mathrm{~A}=$ material constant.

Under loading conditions involving extensive creep and ratcheting deformation, the foregoing treatment can be modified by making a minor revision to Eqn. (2) as detailed below.

Significant plastic deformation occurs at higher temperatures like 823 and $923 \mathrm{~K}$ leading to accumulation of permanent strain through plastic ratcheting through the mean strain acting on the specimen. This imparts a loss of residual ductility in the material. Thus, Eqn. (2) can be revised as follows, incorporating the damage contributions from plastic ratcheting (induced through presence of mean strain) by introducing a parameter $\delta_{c}$ which is the ratcheting strain accumulated per cycle:

$$
\frac{d a}{d N}=A\left(\frac{\Delta \varepsilon_{i n}+\delta_{c}}{D}\right)^{n} a
$$

where $\delta_{c}=$ strain accumulated per cycle through ratcheting, $D=$ material ductility. By integrating Eqn. (3a), a 'ductility normalized equation' can be naturally derived when $\delta_{c}$ is zero, as follows:

$$
\frac{1}{A} \ln \left(\frac{a_{f}}{a_{i}}\right)=\left(\frac{\Delta \varepsilon_{i n}}{D}\right)^{n} N_{f}
$$

where $a_{f}=$ final crack length and $a_{i}=$ initial crack length.

However, for a loading condition where $\delta_{c}>0$, the life prediction equation can be derived from Eqn. (3a) as:

$$
\left(\frac{\Delta \varepsilon_{i n}}{D}\right)^{n} N_{f}=\frac{1}{A}\left(\frac{\Delta \varepsilon_{i n c}}{\Delta \varepsilon_{i n}+\delta_{c}}\right)^{n} \ln \left(\frac{a_{f}}{a_{i}}\right) \equiv \frac{1}{A} \ln \left(\frac{a_{f}}{a_{i, e q i}}\right)
$$


where $a_{i, e q}$ is an equivalent initial crack length incorporating ratcheting as a damage contributor. The crack growth behavior under block loading conditions is presented in Fig. 5, which can be clearly explained in the light of Eqn. 3(c). For uniform representation at all temperatures, crack length is plotted against block-ratio (ratio of the number of blocks at which the crack length is measured to the total number of blocks to failure, designated as $n_{b} / N_{b}$ ). A much higher crack propagation rate at $923 \mathrm{~K}$ compared to the lower temperatures as observed in Fig. 5 may thus be attributed to a higher accumulation of ratcheting strain per cycle. This is also corroborated from the results of the block-loading tests carried out at $923 \mathrm{~K}$ [8, $25]$ on smooth specimens which showed a significant contribution of creep and ratcheting to the net damage $(2.47 \%$ strain accumulation per block). However, at $573 \mathrm{~K}$, contribution of creep and ratcheting becomes almost negligible, with a meager $0.001 \%$ strain accumulation per block $[8,25]$ which explains the significant lowering of crack propagation rate at $573 \mathrm{~K}$ compared to $923 \mathrm{~K}$, in the present case. This is also reflected from a much higher value of the $a_{c r}$ at $573 \mathrm{~K}$ compared to $923 \mathrm{~K}$ (marked by dotted lines in Fig. 5). A similar situation will also occur at $823 \mathrm{~K}$ where significant hardening resulting from dynamic strain aging (DSA) will prevent any loss in residual ductility, thus lowering the contribution of ratcheting to net damage, which is corroborated from the test results carried out under block-loading on smooth specimen with a very low strain accumulation of $0.1 \%$ per block $[8,25]$. It was indicated earlier in $[9,10]$ that DSA (caused by locking of dislocations by $\mathrm{Cr}$ atoms at $823 \mathrm{~K}$ in type 316LN SS [26]) leads to significant hardening of the matrix during cycling which restricts the local plastic deformation associated with the crack tip, thereby leading to a delay in the crack propagation under HCF. This argument was also supported through a slightly higher value of $a_{c r}$ observed at $823 \mathrm{~K}$ in the present case, compared to that of $573 \mathrm{~K}$ (Fig. 5). It is thus clear from the above arguments that the loss of residual ductility due to creep/ratcheting strain accumulation is significantly high at $923 \mathrm{~K}$, resulting in a very high crack growth rate.

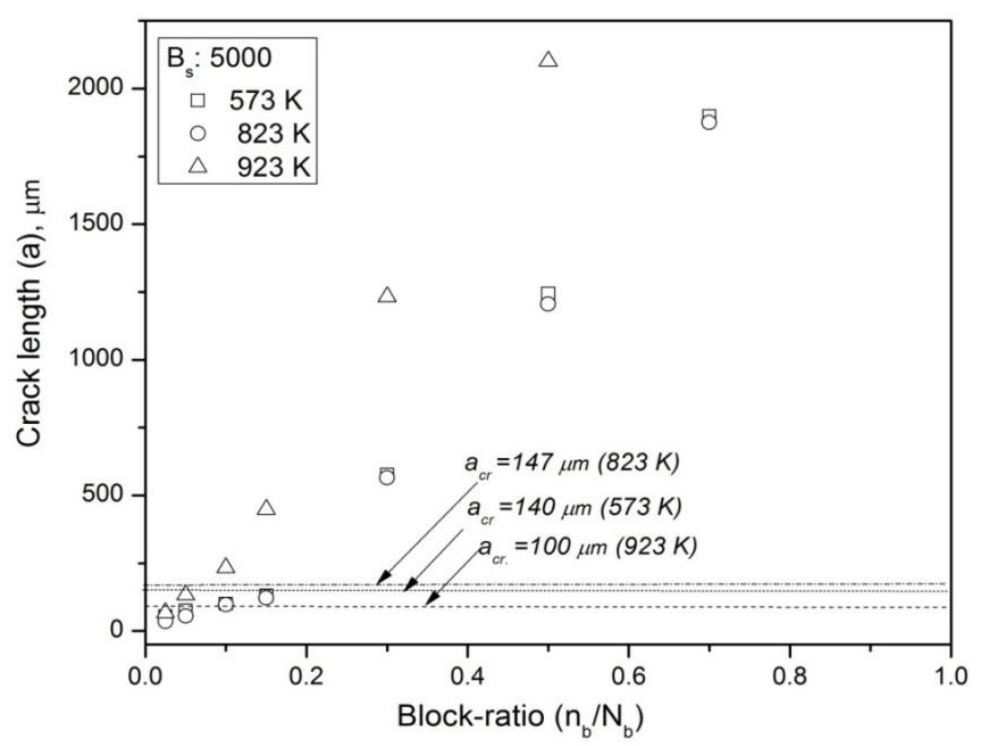

Figure 5: Fatigue crack propagation behavior under strain controlled block-loading (CCF) with $B_{s}: 5000$, T: 573 , 823 and $923 \mathrm{~K}$ $\left(\Delta \varepsilon_{t} / 2_{\mathrm{LCF}}: \pm 0.6 \%\right.$ and $\Delta \varepsilon_{t} / 2_{\mathrm{HCF}}: \pm 0.1 \%$, equivalent $\left.\Delta \sigma_{\mathrm{HCF}}: 25 \mathrm{MPa}\right) . a_{c r}$. marked in the figure indicates the onset of significant LCF$\mathrm{HCF}$ interaction.

$N_{f}$ can be computed from Eqn. 3(c) using the values of $\delta_{c}$ and $D$ for different temperatures once the critical crack length $a_{c r}$ is reached. $a_{c r}$ can be computed in a similar way as shown in section 3.1. However, $a_{c r}$ values will vary with temperature since $\Delta K_{t h}$ from which $a_{c r}$ is computed (Eqn. 1), is a strong function of temperature. In other words, $a_{c r}$ is a characteristic of material mechanics which changes with temperature and loading mode of HCF. Using Eqn. (1), the $a_{c r}$ values in the case of $\Delta \varepsilon_{t} / 2_{\mathrm{HCF}}: \pm 0.1 \%$ (corresponding $\Delta \sigma_{\mathrm{HCF}} \simeq 150 \mathrm{MPa}$ ) were estimated at 140,147 and $100 \mu \mathrm{m}$ respectively, at the temperatures of 573, 823 and $923 \mathrm{~K}$. The estimated values are also found to match fairly well with the experimental results (also marked in Fig. 4) where the crack growth rate is indeed very low.

However, when plastic ratcheting plays a vital role $(923 \mathrm{~K})$, the local plastic strain in the vicinity of the crack increases inspite of the crack length being lower than $a_{c r}$. This in turn, will increase the notch sensitivity leading to early failure at $923 \mathrm{~K}$ compared to that at $573 \mathrm{~K}$. In such cases, it becomes necessary to impose an additional correction factor to account for the damage contribution from creep/ratcheting. One of the ideas is to introduce the correction in terms of 
the ratio of the reduction of crack length in a case with creep/ratcheting (923 K) to a case with no ratcheting (573 K). To obtain this reduction-ratio, $r$, the following method can be used:

$$
\text { Reduction-ratio }\left(r_{c}\right)=\left(a_{f} / a_{c r}\right)_{\text {ratcheting }} /\left(a_{f} / a_{c r}\right)_{n o} \text { ratcheting }
$$

The value of $r_{c}$ is calculated as $r_{c}=0.71$ under the present experimental condition using the $a_{c r}$ values as mentioned above. Hence, the modified value of $a_{c r}$, designated as $a_{c, r, q}$ can be computed at $923 \mathrm{~K}$ as follows:

$$
a_{c r, e q .}=0.71 * a_{c r}=71 \mu \mathrm{m}(923 \mathrm{~K})
$$

Thus, $a_{c r, e q}$. is a common crack size independent of temperature and $B_{s}$ which takes into account the effect of plastic ratcheting at $923 \mathrm{~K}$.

$$
a_{c r, e q}=r_{c}^{*} a_{c r}
$$

At ambient temperature or $823 \mathrm{~K}$, effect of plastic ratcheting is negligible and $r_{c}$ remains 1 , indicating that in such cases, $a_{c r, e q}=a_{c r}$.

Since $a_{c, \text { req. }}$. marks the onset of crack propagation at $923 \mathrm{~K}$, it must be an important parameter to avoid the occurrence of ratcheting under block-loadings, since ratcheting will not play a dominant role under block-loading when a $<a_{c r, e q}$. Hence, at $923 \mathrm{~K}$, the crack should be limited to this value (marked in Fig. 6) to avoid the damaging consequences of creep and ratcheting. However, it may be noted that the term $a_{c r, e q}$ primarily addresses the effect of plastic ratcheting and not creepinduced ratcheting for which separate equation needs to be used.

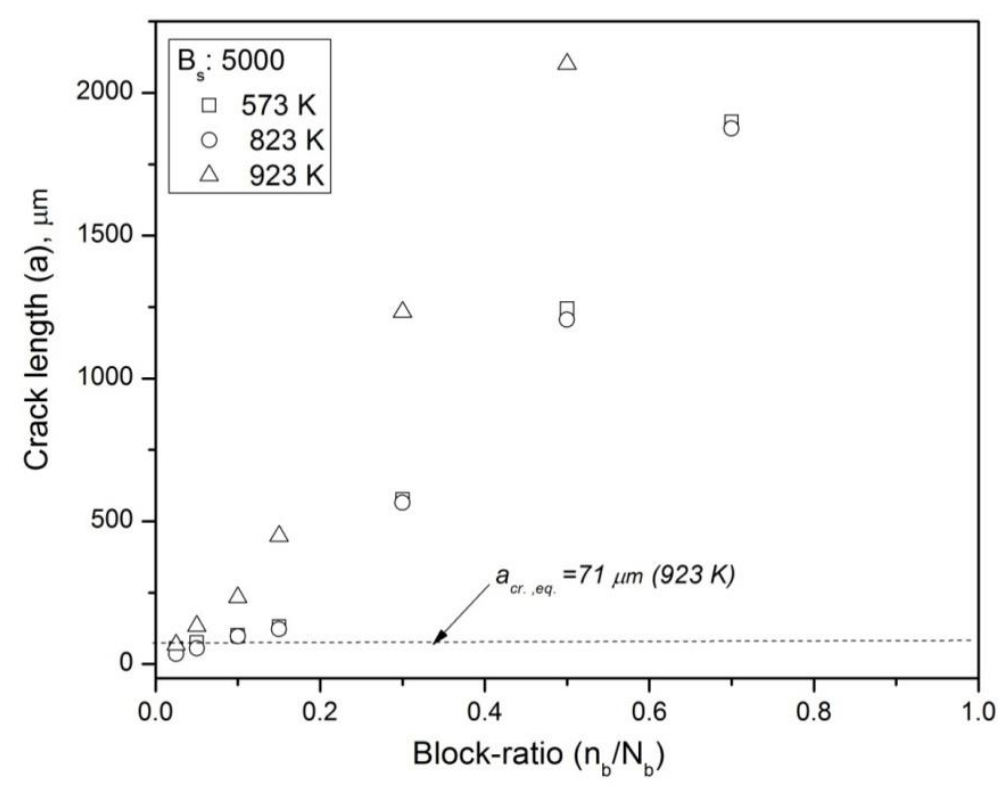

Figure 6: Fatigue crack propagation behavior under strain-controlled block-loading (CCF) with $B_{s}: 5000$, T: 573 , 823 and $923 \mathrm{~K}$ $\left(\Delta \varepsilon_{t} / 2_{L C F}: \pm 0.6 \%\right.$ and $\Delta \varepsilon_{t} / 2_{H C F}: \pm 0.1 \%$, equivalent $\left.\Delta \sigma_{H C F}: 25 \mathrm{MPa}\right) . a_{c}$ is replaced by $a_{c r, e q}$ at $923 \mathrm{~K}$ to account for creep/ratcheting damage (marked in the figure by dotted line)

It is clear from the above discussion that Eqn. 3 (c) is primarily based on crack propagation under ratcheting in a blockloading condition and hence can be used for predicting the remaining life at $923 \mathrm{~K}$ only when $a_{c r, e q}$ is reached. Similarly, Eqn. 3(b) can be used for remaining life spent in crack propagation for other temperatures like 573/823 $\mathrm{K}$ where ratcheting is insignificant, only when $a_{c r}$ is reached. Hence, life-estimation under LCF-HCF loadings follow a two-step procedure (1) Estimation of $a_{c r}$ or $a_{c r, e q}$ using Eqn. 1 or Eqn. 4 (2) Estimation of the life spent in crack propagation using Eqn. 3(b) /Eqn. 3 (c). Hence, the final life-prediction equation is modified as follows: 


$$
\begin{array}{ll}
\left(\frac{\Delta \varepsilon}{D}\right)^{n} N_{f} \equiv \frac{1}{A} \ln \left(\frac{a_{f}}{a_{c r}}\right) & \text { (for no ratcheting) } \\
\left(\frac{\Delta \varepsilon}{D}\right)^{n} N_{f} \equiv \frac{1}{A} \ln \left(\frac{a_{f}}{a_{c r, e q .}}\right) & \text { (for ratcheting) }
\end{array}
$$

Thus, Eqn. 5 and 6, based on crack propagation may be treated as a universal equation which can be used for lifeprediction under LCF-HCF interaction irrespective of the temperature, using suitable values of $a_{c r}$ or $a_{c r, e q}$. It may be noted that changes in crack dynamics due to the accumulation of strain through ratcheting are adequately accounted for, in the above equation.

In real-life, crack-sizes can be measured at elevated temperature online using NDT techniques like acoustic emission technique. However, in the present case, critical crack-length $\left(a_{c r}\right)$ was estimated using fatigue crack threshold, $\Delta \mathrm{K}_{\text {th }}$ from literature.

The procedure for life-estimation for any arbitrary loading sequence consisting of LCF and HCF loads can be expressed through an algorithm (Fig. 7) where effect of plastic ratcheting at $923 \mathrm{~K}$ or effect of cyclic hardening due to DSA is accounted for incorporating material ductility as an important parameter. Life-prediction was carried out based on this algorithm using Eqn. 5 and 6, and the tabulated against experimental life in Tab. 1. Here, the values of 'A' and ' $n$ ' used in the equations are based on previous experiments on 316L SS [20], and the value of 'D' is used from the tensile data on 316LN SS [27]. A fairly good estimation was found against experimental life, with $\sim 80 \%$ accuracy. It is also clear that usage of correction factor results in a better estimation in a case where ratcheting/creep is strongly prevalent (as in 923 $\mathrm{K})$. The estimation can be further improved by incorporating more data on strain controlled block-loading experiments at different temperatures.

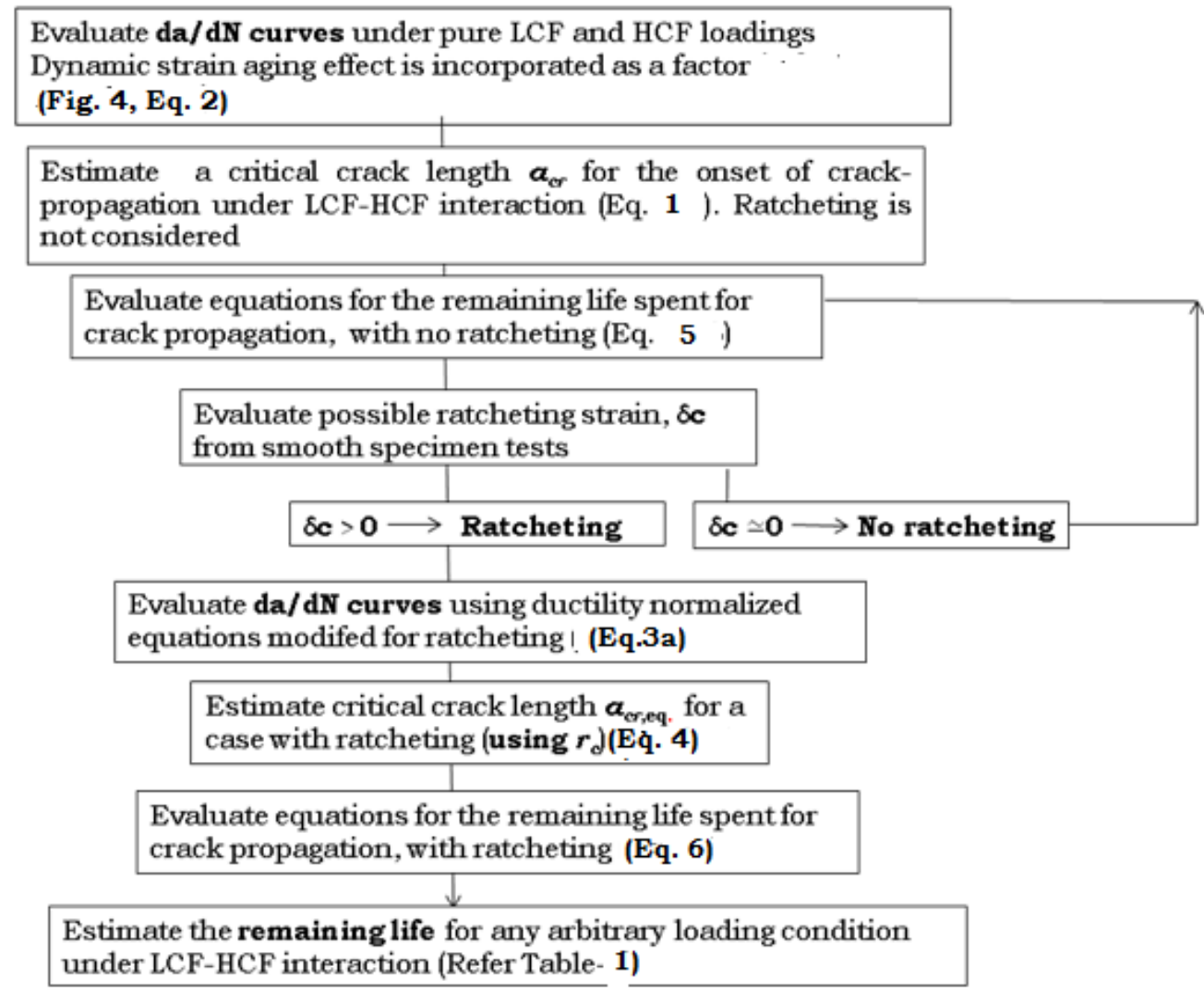

Figure 7: Algorithm depicting the procedure of life-estimation under LCF-HCF interaction for any arbitrary loading sequence

In absence of smooth specimen ratcheting data, simulation of ratcheting behavior can be carried out using ArmstrongFrederick non-linear kinematic hardening model or Ohno-Wang model [28-29]. However, such models should be refined in the present case incorporating material ductility as an important parameter to account for the effect of cyclic hardening 
or temperature (effect of DSA) for predicting ratcheting strain more accurately, particularly in the DSA temperature regime. Although the universal equation based on the concept of fracture mechanics can able to predict life in a much better way compared to the modified DCA model, the equation is better suited for fatigue (cycle dependent damage) and hence can also address the issue of plastic ratcheting. It may also be noted that the model is developed based on the assumption that crack nucleates from the surface of the specimen. From a practical point of view, the LCF crack is expected to nucleate on the surface of the inner vessel of the reactor facing the sodium which may be further enhanced by presence of HCF damage/creep or creep-assisted ratcheting damage during the steady state operation. However, initiation of crack from the internal (bulk) cannot be ruled out due to the presence of strong ratcheting and creep damage. In such a case, the present model may not be able to predict life accurately. This envisages the need of refining the present model by using separate constitutive equations based on time dependent creep damage ( $d a / d t)$ rather than cycle dependent damage.

\begin{tabular}{ccccc}
\hline Temperature & $\begin{array}{c}\text { Estimated } \\
\text { Life }\end{array}$ & $\begin{array}{c}\text { Experimental- } \\
\text { life }\end{array}$ & $a_{\text {cr }}$ & Accuracy(\%) \\
573 & 5580 & 6400 & 140 & 82 \\
823 & 3700 & 4230 & 147 & 85 \\
923 & 710 & 287 & 100 & - \\
& 327 & & $71^{*}$ & 83 \\
\hline
\end{tabular}

Table1: Predicted values of life under LCF-HCF interaction as per the algorithm ("indicates $a_{c r}$ value using correction factor for creep/ratcheting).

\section{CONCLUSIONS}

A $\mathrm{n}$ attempt was made towards remaining life-estimation under LCF-HCF interaction in block-loading sequence using the concept of a critical crack-length $\left(a_{c r}\right)$, estimated using fatigue crack threshold, $\Delta K_{t b}$. A unified approach was developed and extended towards remaining life-estimation using $a_{c r}$. The model was also able to address the influence of DSA, creep and ratcheting in prediction of remaining life.

\section{REFERENCES}

[1] Bhoje, S.B. and Chellapandi, P. (2000). Advanced Structural design of high temperature components of FBR, Trans. Indian Inst. Metals, 71, pp. 165-173.

[2] Halford, G.R. (1997). Cumulative fatigue damage modeling — crack nucleation and early growth, Int. J. Fatigue, 19, pp. S253-S260.

[3] Wong, Y.K., Hu, X.Z. and Norton, M.P. (2002). Plastically elastically dominant fatigue interaction in 316L stainless steel and 6061-T6 aluminium alloy, Fatigue Fract. Eng. Mater. Struct., 25, pp. 201-213.

[4] Wong, Y.K., Hu, X.Z. and Norton, M.P. (2002). An alternative definition for two distinct regions of fatigue, Proc. Struct. Integrity and Fract. Conf., USA

[5] Manson, S.S. and Halford, G.R. (1981). Practical implementation of the double linear damage rule and damage curve approach for treating cumulative fatigue damage, Int. J. Fract., 17, pp. 169-192

[6] Suresh, S. (1991) Fatigue of materials, Cambridge university press, UK

[7] Dieter, G.E. (1988) Mechanical Metallurgy, McGraw-Hill Book Company, UK

[8] Sarkar, A., Okazaki, M., Nagesha, A., Sandhya, R., Parameswaran, P. and Laha, K. (2016). Mechanisms of failure under low cycle fatigue, high cycle fatigue and creep interactions in combined cycling in a type 316LN stainless steel, Mater. Sci. Eng: A., 683, pp. 24-36

[9] Neeharika, V. B., Narayana, K. S., Krishna V., and. Kumar, M. P. (2014) . Tensile and creep data of 316LN stainless steel ananlysis, ARPN J. Eng. Appl. Sci., 9, pp. 699-705

[10] Sarkar, A., Nagesha, A., Sandhya, R., Laha, K. and Okazaki, M. (2018). Manifestations of dynamic strain aging under low and high cycle fatigue in a type 316LN stainless steel, Mater. High Temp., 35(6), pp. 523-528

[11] Sarkar A, Ph.D thesis (2018). Low Cycle Fatigue-High Cycle Fatigue Interaction in Type 316LN Stainless Steel at High Temperatures, Nagaoka University of Technology, Nagaoka, Japan. 
[12] Sarkar, A., Nagesha, A., Sandhya, R., Parameswaran, P., Laha and K., Okazaki, M. (2017). Investigation of cumulative fatigue damage through sequential Low cycle Fatigue and High cycle Fatigue cycling at high temperature for a type 316LN stainless steel: Life-Prediction techniques and associated mechanisms, Metall. Mater. Trans A., 48 (3), pp. 953964.

[13] Sarkar, A., Nagesha, A., Sandhya, R., Parameswaran, P., Laha and K., Okazaki, M. (2017). Investigation of fracture mechanisms and substructural changes under sequential fatigue cycling involving LCF and HCF loads in a type 316LN stainless steel at 923 K. Mater. Sci. Eng: A, 702, pp.360-370

[14] McDowell, D.L. (1996) Basic issues in the mechanics of high cycle metal fatigue, Int. J. Fract., 80, pp. 103-145.

[15] Samuel, K.G., Sasikala, G. and Ray, S.K. (2011). On R ratio dependence of threshold stress intensity factor range for fatigue crack growth in type 316(N) stainless steel weld, Mater. Sci. Tech., 27(1), pp. 371-376.

[16] Huthman, H., Livesy, V.B. and Robert, G. (1996) Fatigue crack growth threshold and short crack growth in austenitic materials, Int. J. Press. Vess. Pip., 65, pp. 231-239.

[17] Okazaki, M., Sawada, T., Kasahara N. and Kamaya, M. (2011). An Investigation on High-Cycle Thermal Fatigue Failure Based on Crack Propagation in Type 316 Stainless Steel, Structural Safety and Reliability (JCOSSAR 2011), pp. 127-135.

[18] ASTM Standards (2003). E647-00, Vol. 03.01, ASTM International, p. 615.

[19] Shih, C.F. and Hutchinson, J.W. (1976). Fully plastic solutions and large scale yielding estimates for plane stress crack problems, Trans. ASME, J. Eng. Mater. Tech..98, p.289.

[20] Miyahara, M. and Tokimasa, K. (1986). High temperature fatigue properties and life prediction of SUS 304 stainless steel under variable straining, Trans. Soc. Mater. Science Japan, 35, pp. 1030-1036.

[21] Dowling, N. E., and. Begley, J. A. (1976) Fatigue crack growth during gross plasticity and the J-integral, In Mechanics of crack growth. ASTM International.

[22] El Haddad, M. H., Smith, K. N and Topper., T. H. (1979) Fatigue crack propagation of short cracks, Journal of Engineering Materials and Technology, 101(1), pp. 42-46.

[23] Starkey, M.S. and Skelton, R.P. (1982). A comparison of the strain intensity and cyclic J approaches to crack growth, Fatigue Fract. Eng. Mater. Struct., 5(4), pp.329-341.

[24] Haigh, J.R. and Skelton, R.P. (1978) A strain intensity approach to high temperature fatigue crack growth and failure, Mater. Sci. Eng. A 36, pp. 133-137.

[25] Sarkar, A., Nagesha, A., Parameswaran, P., Sandhya, R., Laha, K. and Okazaki, M. (2017). Evolution of damage under combined low and high cycle fatigue loading in a type 316LN stainless steel at different temperatures, Int. J. Fatigue, 103, pp. 28-38.

[26] Mannan, S.L. (1993). Role of dynamic strain ageing in low cycle fatigue, Bull. Mater. Sci., 16(6), pp. 561-582.

[27] Ganesh Kumar, J., Chowdary, M., Ganesan, V., Paretkar, R.K., Rao, K.B.S., Mathew, M.D. (2010). High temperature design curves for high nitrogen grades of 316LN stainless steel, Nucl. Eng. Des., 240, pp. 1363-1370.

[28] Armstrong, P. J. and Frederick, C.O. (1966) Report RD/B/N73, Central Electricity Generation Board, Berkley Nuclear Laboratories, Berkley, UK.

[29] Ohno, N. and Wang, J.D. (1993) Kinematic Hardening Rules With Critical State of Dynamic Recovery: Part IFormulation and Basic Features for Ratcheting Behavior, Int. J. Plast., 9, pp. 375-390.

\section{NOMENCLATURE}

$\varepsilon_{\text {in }} \quad$ Inelastic strain

$\Delta \varepsilon_{t} / 2 \quad$ LCF strain amplitude

$\sigma_{\text {LCF }} \quad$ LCF stress amplitude

$\sigma_{\text {HCF }} \quad$ HCF stress amplitude

$B_{s} \quad$ Block-size

$N_{f} \quad$ Number of cycles to failure

$\Delta K_{t h} \quad$ Threshold stress intensity factor

$\Delta K \quad$ Range of the stress intensity factor

$\Delta J \quad$ Strain energy release rate

a Crack length

$a_{c r} \quad$ Critical crack length

$F \quad$ Boundary correction factor associated with stress intensity factor 


\begin{tabular}{ll}
$d a / d N$ & \multicolumn{1}{c}{ Crack growth rate } \\
$\delta_{c}$ & Ratchet/creep strain accumulated per cycle \\
$D$ & Material ductility \\
$a_{f}$ & Final crack length \\
$a_{0}$ & Initial crack length \\
$a_{i}$ & Crack length at ith cycle \\
$r_{c}$ & Reduction-ratio of crack length \\
$a_{i, e q}$ & Equivalent crack length \\
$a_{c r, e q}$ & Equivalent critical crack length \\
$A, n$ & Material constants in crack growth equation
\end{tabular}

